\title{
New Theory CVD \Cancer Explain the Mechanism of Heart Failure
}

\author{
Ermoshkin Vladimir Ivanovich* \\ Russian New University (RosNOU), Russia
}

*Corresponding author: Ermoshkin Vladimir Ivanovich, Russian New University (RosNOU), 105005, Moscow, Radio street, 22, Russia

\begin{tabular}{|c|c|}
\hline ARTICLE INFO & ABSTRACT \\
\hline Received: 慧 October 22, 2019 & Citation: Ermoshkin Vladimir Ivanovich. New Theory CVD \Cancer Explain the \\
\hline Published: 㓞 October 30, 2019 & Mechanism of Heart Failure. Biomed J Sci \& Tech Res 22(3)-2019. BJSTR. MS.ID.003742. \\
\hline
\end{tabular}

\section{Purpose}

Cardiovascular disease and cancer are the leading causes of death worldwide. Huge amounts of money are invested in the research of medicine, but so far, the successes of cardiology and Oncology are insufficient. Let's once again pay attention to the proposals of the New Theory of CVD $\backslash$ cancer.

\section{Method}

The study of numerous sources of information posted on the Internet. Discussion of proposed ideas at conferences. Discussion of new ideas with leading cardiologists.

\section{Results}

In the opinion of the author of "New theories CVD \cancer" weak result happens, including the strategic scientific errors of medical management. Modern medicine has divided medical theory and practice into separate areas in which each disease and each organ was treated by separate specialists. In addition, there seems to have been little involvement of physicists, mathematicians, chemists in the discussion of major medical problems. Little attention was paid to the development of integral medicine. The new theory of CVD \ cancer [1-10], since 2011, tries to prove that in healthy from birth people, many diseases are caused by the non-optimal operations of large arteriovenous anastomoses (AVA). This is a completely new mechanism of CVD; it should be discussed. Stress, lack of exercise, overeating, unhealthy lifestyle can increase arterial blood pressure (AP) at some periods. To artery not damaged from a sprain, in periods of increasing AP open AVA [11] and part of the arterial blood goes directly into the vein. This leads to a sharp decrease in arterial blood pressure, there may be jumps in AP.
Note that this optimization of AP leads to a disproportion in the volume of venous and arterial blood. An increase in venous volume taking into account gravity forces and body position leads to an increase in venous blood pressure (VBP), stagnation of blood and other fluids in the veins, arteries, intercellular space, lymphatic vessels. Confirmation: enlargement and fibrosis of the liver, formation of a pattern of «jellyfish» on the abdomen and varicose veins on the legs. In parallel, different types of arrhythmia may occur. This is due to the overflow of venous blood system in portal veins of the liver, hollow veins, the entire venous pool. The mechanism of arrhythmia is most often the occurrence of a superposition of two types of myocardial excitation, using bioelectric and mechanical pulses (pulse of heart). For example, trigeminy is an alternation of types of excitation: two excitations from the sinus node (narrow waves), the third because of the concentration of mechanical energy on the apex of the heart (wide wave), etc.

The equal distance between the QRS teeth in paroxysmal tachycardia is due to the equal time of travels the mechanical wave along the contour of the vessels: aorta, liver, hollow vein, myocardium. Cardiopathy and arteriosclerosis of vessels further increase the range of possible arrhythmias, including fibrillation may occur. Over time, due to the regular increase in VBP, a chain of pathological events may occur damage to venous valves, venous fullness, due to insufficient pressure difference between arterioles and venules in cells, malnutrition, ischemia of organs, thrombosis of small vessels in the lower half of the body. Groups of cells subject to ischemia, necrosis, apoptosis and not removed from the body in time - this is the environment for the possible development of cancer. 
The cause of comorbidity of diseases, thanks to the New Theory, becomes natural, because there can be no other way with a long increase in venous pressure. Mechanism one, but many diseases. This explains the pairwise correlation of many CVD and cancer. And official medicine still every year explores the relationships, finds significant correlations between any diseases and is very surprised by his discoveries.

Later, over some years, usually in the supine position during sleep, significant amounts of fluids each night begin to flow towards the head. Excessive venous pressure triggers pathological changes in the upper half of the body: there is a delay in venous blood in the lungs, slowing the outflow of venous blood from the brain, morning headaches, etc. In addition, thanks to the new theory, there is an explanation for the increase in blood sugar in diabetics 4-6 hours after the start of a night's sleep. This is due to the movement of stagnant during the day in the lower half of the body venous blood towards the upper half. Faster dirty blood out of the capillaries and small vessels cannot. And even in healthy people there is a small jump in glucose at dawn or the effect of «morning dawn». But official medicine does not notice all these signs!

\section{Summary}

It is very likely that unnoticed by doctors «venous fullness», leading to so-called «heart failure» and ischemia of organs, leads to many pathologies of CVD and other organs, leads to deterioration of human health and high mortality. We need to find methods to influence improperly functioning anastomoses AVA. It is necessary to study this problem. It is necessary to recognize some of the principles of the New Theory of CVD \cancer and, apparently, to begin to revise the theoretical issues in medicine.

\section{ISSN: 2574-1241}

DOI: 10.26717/BJSTR.2019.22.003742

Ermoshkin Vladimir Ivanovich. Biomed J Sci \& Tech Res

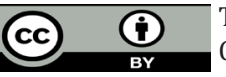

This work is licensed under Creative Commons Attribution 4.0 License

Submission Link: https://biomedres.us/submit-manuscript.php

\section{Biography}

Vladimir Ermoshkin completed his Graduation in Physics department at Moscow State University in 1978. He has worked at Russian New University (RosNOU) as Physicist. He has published about 20 articles on Cardiology in prominent magazines. Since 2012, V. Ermoshkin have participated in several international medical conferences.

\section{References}

1. Ermoshkin VI (2016) New theory of arrhythmia. Conceptual substantiation of arrhythmia mechanisms. Cardiometry 8: 6-17.

2. Ermoshkin VI (2016) A New Theory of Certain Cardiovascular Diseases. EC Cardiology 2(5).

3. Ermoshkin V (2016) Pathological Role of the "Invisible" Anastomoses. J Bioengineer \& Biomedical Sci 6: 209.

4. Ermoshkin VI (2016) Proceedings of the International Conference on arrhythmia. Australia. Brisbane. Arrhythmia.

5. Kamkin AG, Kiseleva IS, Yarygin VN (2002) Fibrillation, defibrillation. Priroda 4: 1040.

6. Ermoshkin VI (2017) Problems of heart failure. Unexpected outcome. London 15-17.

7. Kaur J (2014) A comprehensive review on metabolic syndrome. Abstract.

8. Grundy SM (2007) Metabolic syndrome: a multiplex cardiovascular risk factor. J Clin Endocrinol Metab 92(2): 399-404.

9. Ermoshkin Vladimir Ivanovich (2019) Fecal Transplantation to Restore a Healthy Intestinal Microbiota. An Explanation of the Effectiveness of this Procedure is Given by the New Theory of CVD and Cancer. EC Cardiology 8: 715-721.

10. Ermoshkin Vladimir Ivanovich (2019) The Cause of Inflammatory Bowel Disease - Increased Venous Pressure in the Lower Half of the Human Body. EC Cardiology 6(3): 231-234.

11. Internet resource, Clinical case. Russian. 